\title{
Correction: India's energy subsidy slowdown
}

Lucas Laursen

Nature Energy 1, 16056 (2016); published 9 May 2016; corrected 23 May 2016.

In the version of this Feature originally published, the name of the individual introduced at the beginning was incorrect throughout and should have read Gurumoorthy Sethuraman. 